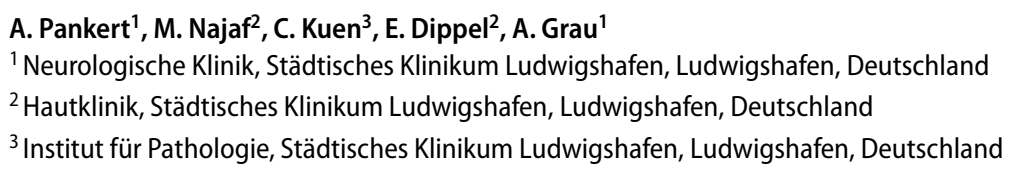

\title{
Papeln und Kopfschmerz - wie passt das zusammen?
}

\section{Anamnese}

Eine 42-jährige osteuropäische Patientin stellte sich aufgrund von seit 2 Tagen bestehenden Kopfschmerzen drückenden Charakters und hoher Intensität vor, die sie als beidseits vom Nacken aus über den Hinterkopf nach frontal ausstrahlend beschrieb. Eine symptomatische Therapie erbrachte keinen Erfolg. Zudem bestand seit 3 Tagen ein Exanthem an den Armen und am Dekolleté. Im Monat zuvor habe sie einen Insektenstich am Unterschenkel gehabt, der sich infiziert habe und mit einer Lymphknotenschwellung einhergegangen sei. Ambulant sei eine antibiotische Therapie mit Amoxicillin über 5 Tage durchgeführt worden. Seit dem Vortag der Aufnahme habe sie Fieber bis $39,1^{\circ} \mathrm{C}$. Seit 3 Wochen leide sie an Husten, den sie auf einen bekannten Heuschnupfen zurückführe.

\section{Erste Befunde}

Der neurologische Untersuchungsbefund war bis auf einen Meningismus unauffällig. In der körperlichen Untersuchung fanden sich insbesondere im Bereich der Arme und des Dekolletés (Abb. 1), aber auch im Bereich des Rückens und Gesichts Papeln und urtikarielle Plaques (Abb. 2).

Die Aufnahme erfolgte bei unklarem Fieber und Husten, da die Vorstellung in den Pandemiezeitraum fiel, zunächst auf die COVID-19-Verdachtsstation. Nach negativem Abstrich und unauffälliger hochauflösender Computertomografie (CT) des Thorax diesbezüglich erfolgte die neurologische Übernahme. Im Aufnahmelabor zeigten sich bis auf eine Eisenmangelanämie und CRP-Erhöhung von 60,6 mg/dl keine Auffälligkeiten. Die kraniale Computer- und Magnetresonanztomographie fielen unauffällig aus. In der Liquoruntersuchung zeigte sich eine Pleozytose von 18 Zellen/ $\mu$ l bei überwiegend mononukleärem (73\%) Zellbild. Eiweiß, Glukose und Lactat waren sämtlich normwertig. Die Herpes- (EBV-, VZV-, HSV-, CMV-), Respiratorisches Syncytial-Virus(RSV)- und Influenza-PCR sowie die Serum-/Liquordiagnostik im Hinblick auf Borrelien, Lues, $M y$ coplasma pneumoniae, Treponema pallidum, Frühsommer-Meningoenzephalitis (FSME), Masern, Mumps und Röteln zeigten sich ebenfalls unauffällig.

\section{Redaktion}

J. Bösel, Kassel

S. Schönenberger, Heidelberg

DGNeurologie 2020 · 3 (5): 390-394

https://doi.org/10.1007/s42451-020-00221-6

Online publiziert: 14. August 2020

(c) Springer Medizin Verlag GmbH, ein Teil von Springer Nature 2020

\section{Weitere Befunde und Verlauf}

Aufgrund der Hautveränderungen erfolgte eine dermatologische Vorstellung. Seitens der Kollegen wurde der Verdacht auf ein Sweet-Syndrom erhoben. Es wurden mehrere Biopsien entnommen, anhand derer die Verdachtsdiagnose bestätigt wurde (Abb. 3a, b).

Aufgrund der aseptischen Meningitis im zeitlichen Zusammenhang gingen wir von einem Neuro-Sweet-Syndrom aus. Bis zum Erhalt der negativen Herpes-PCR erfolgte eine Aciclovirtherapie, die dann rasch beendet wurde.

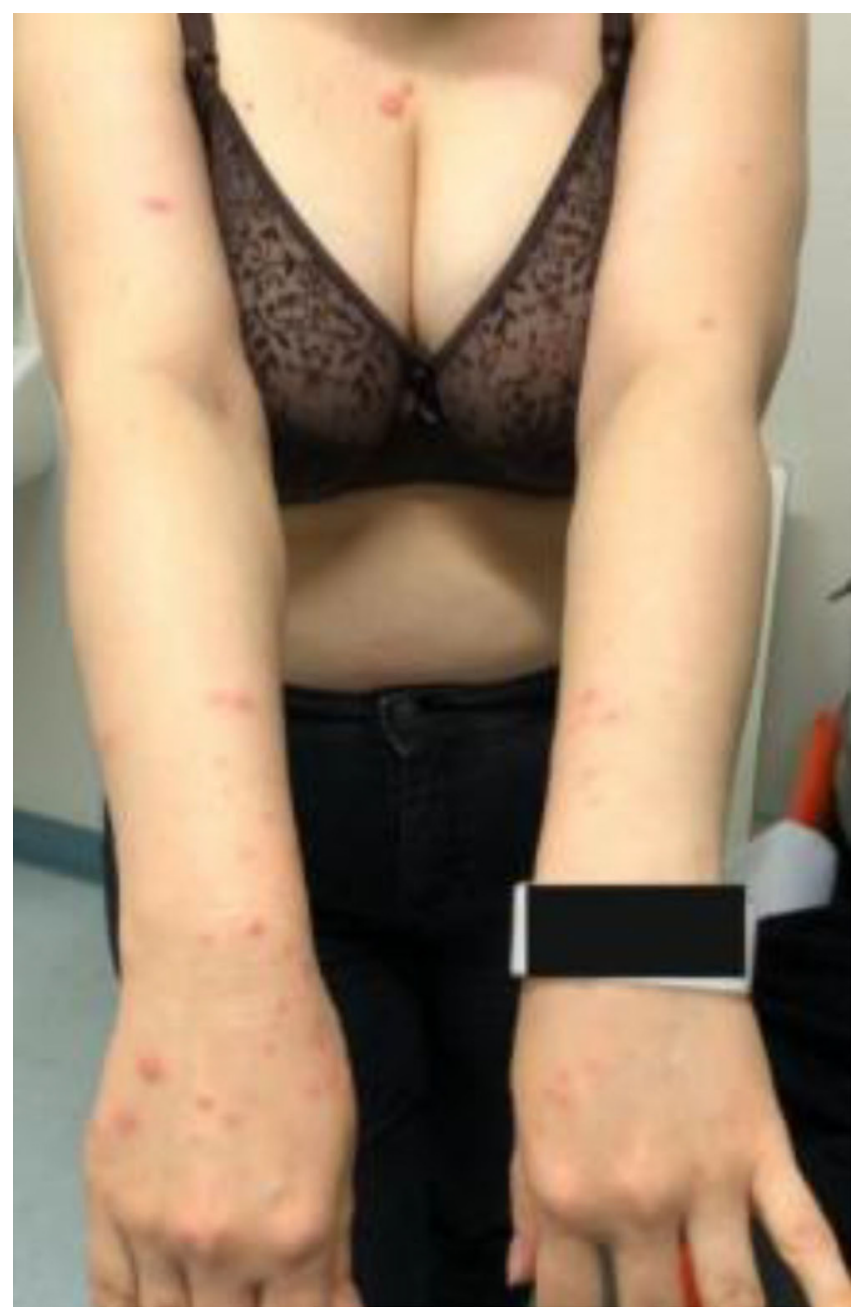

Abb. 1 Papulöses Exanthem vorwiegend im Bereich der Arme/des Dekolletés 

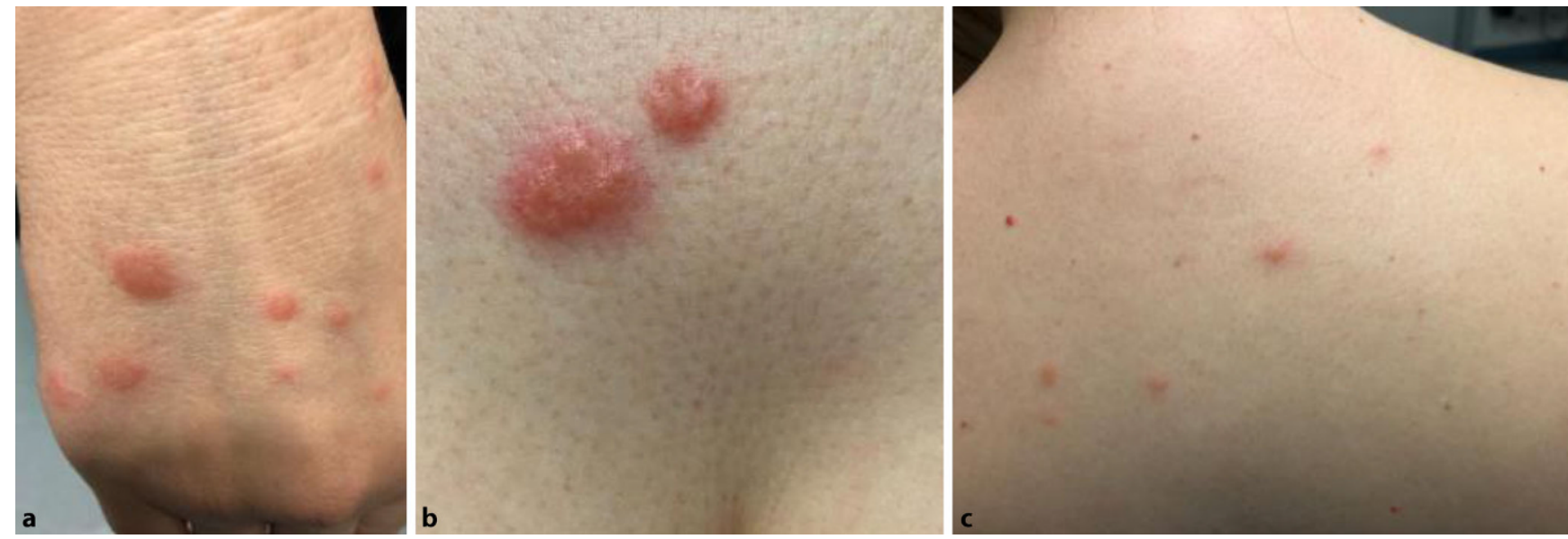

Abb. 2 Papeln im Bereich der Hand (a), des Dekolletés (b) und des Rückens (c)
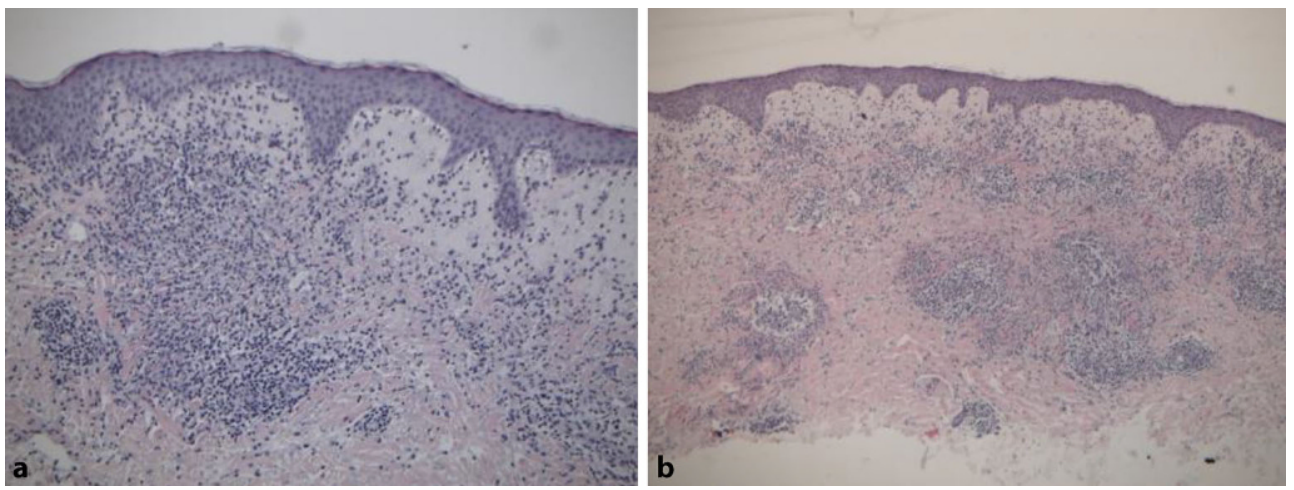

Abb. 3 Akute neutrophile Dermatose mit herdförmigem dichtem dermalem Infiltrat aus (überwiegend stabkernigen) neutrophilen Granulozyten mit teilweise Leukozytoklasie und deutlichem Begleitödem

Wenige Tage nach der Aufnahme gab die Patientin komplette Beschwerdefreiheit an und wünschte aus dringenden häuslichen Gründen eine Entlassung vor den anstehenden Osterfeiertagen, sodass wir von einer Glukokortikoidtherapie absahen, die weiter unten aufgeführte Diagnostik nicht komplettieren konnten und dem Hausarzt empfahlen, diese zu ergänzen. Da hierdurch von augenärztlicher Seite keine Uveitis ausgeschlossen werden konnte, waren zu jenem Zeitpunkt lediglich die Kriterien für ein mögliches Neuro-Sweet-Syndrom erfüllt (Tab. 2).

\section{Diskussion}

Aseptische Meningitiden können vielseitige Ursachen haben und stellen eine diagnostische Herausforderung dar. Neben viralen Infektionen kommen zahlreiche nichtinfektiologische Differenzialdiagnosen (Tab. 1 und 3) in Frage.

Neben dem geschilderten Neuro-Sweet-Syndrom sollte bei aseptischer Meningitis mit Hautläsionen insbesondere auch an einen Neuro-Behçet gedacht werden, der viele Ähnlichkeiten aufweist.

Das Sweet-Syndrom wurde erstmals von Dr. Robert Douglas Sweet [10] im Jahr 1964 beschrieben. Es handelt sich hierbei um eine Multisystemerkrankung unklarer Ätiologie, die durch Fieber, Unwohlsein, eine Leukozytose und erythematöse Hautläsionen gekennzeichnet ist, die v. a. Gesicht, Rumpf und obere Extremitäten betreffen und ohne Narbenbildung abheilen. Die Erkrankung kann eine idiopathische Genese haben oder tumoroder medikamentenassoziiert auftreten [2]. Die idiopathische
Form betrifft vorwiegend weibliche Personen zwischen dem 30 . und 60. Lebensjahr. Sie kann mit respiratorischen und gastrointestinalen Infektionen assoziiert sein und tritt auch während der Schwangerschaft auf.

Die tumorassoziierte Form wird mit hämatoonkologischen Erkrankungen wie der akuten myeloischen Leukämie sowie soliden Organtumoren in Verbindung gebracht. Zudem können zahlreiche Medikamente, v. a. der Granulozyten-koloniestimulierende Faktor (GCSF), ein Sweet-Syndrom auslösen. Der Erkrankung geht oft eine 1-3 Wochen zuvor aufgetretene bakterielle Infektion voraus [8]. Neben der Affektion der Haut kann es auch zu anderen Organmanifestationen (Augen, Lunge, Leber, Nieren, Gastrointestinaltrakt, Knochenmark, Muskeln und ZNS) kommen [7].

Das Neuro-Sweet-Syndrom wurde erstmals im Jahr 1999 durch Hisanaga et al. [6] beschrieben. Es handelt sich um ein seltenes Krankheitsbild, das v. a. asiatische Patienten betrifft. Die häufigste in der Literatur beschriebene neurologische Manifestation ist die (Meningo-)Enzephalitis. Unter Symptomen werden Kopfschmerzen, Bewusstseinsstörungen, epileptische Anfälle, Paresen, Gedächtnisstörungen und psychiatrische Störungen aufgeführt.

Meist gehen die typischen Hautveränderungen der neurologischen Symptomatik voraus. Ein gleichzeitiges Auftreten dermatologischer und neurologischer Symptome oder ein Vorausgehen der neurologischen Symptome sind aber ebenfalls vorbeschrieben. Laborchemisch finden sich häufig eine CRP- und 
Tab. 1 Hauptsächlich mit aseptischer Meningitis assozilerte Systemerkrankungen. (Nach Tattevin et al. [11])

\begin{tabular}{|c|c|c|c|}
\hline Krankheitsbild & Epidemiologie & Klinik & Liquorbefund \\
\hline Sarkoidose & $\begin{array}{l}\text { w>m } \\
\text { Alter 20-40 } \\
\text { Höhere Prävalenz in der } \\
\text { Sub-Sahara-Region sowie in } \\
\text { der Karibik }\end{array}$ & $\begin{array}{l}\text { Neurologische Symptome (Hirnnervenparesen, chronische Menin- } \\
\text { gitiden) oft bereits in der Frühphase der Erkrankung } \\
\text { Nichtneurologisch: vergrößerte Lymphknoten, Parotitis, Uveitis, Erythe- } \\
\text { ma nodosum, Arthritis } \\
\text { Nachweis über Granulome in Speicheldrüsenbiopsien oder Biopsaten } \\
\text { anderer betroffener Regionen (z. B. Haut) }\end{array}$ & $\begin{array}{l}\text { Lymphozytäre Pleo- } \\
\text { zytose } \\
\text { Eiweißerhöhung } \\
\text { Normoglykämie }\end{array}$ \\
\hline $\begin{array}{l}\text { Sjögren- } \\
\text { Syndrom }\end{array}$ & $\begin{array}{l}\text { Vorwiegend weibliche } \\
(90 \%) \text { Personen } \\
\text { Auftreten typischerweise } \\
\text { nach dem } 40 \text {. Lebensjahr }\end{array}$ & $\begin{array}{l}\text { Neurologisch: Kombination peripher- (Polyneuropathie, Hirnnervenläh- } \\
\text { mungen) und zentralnervöser Läsionen } \\
\text { Nichtneurologisch: Sicca-Symptomatik, Anti-SSA (Ro), Anti-SSB (La), } \\
\text { Diagnosestellung zusätzlich über Speicheldrüsenbiopsat }\end{array}$ & $\begin{array}{l}\text { Lymphozytäre Pleo- } \\
\text { zytose, normal bis } \\
50 / \mu l[9] \text {, mit Plasma- } \\
\text { zellen }\end{array}$ \\
\hline $\begin{array}{l}\text { Systemischer Lupus } \\
\text { erythematodes }\end{array}$ & $\begin{array}{l}\text { Vorwiegend weibliche Per- } \\
\text { sonen } \\
\text { Auftreten vorwiegend vor } \\
\text { dem 40. Lebensjahr } \\
\text { Positive Familienanamnese } \\
\text { für Autoimmunerkrankun- } \\
\text { gen }\end{array}$ & $\begin{array}{l}\text { Neuropsychiatrisch: Depression, Psychosen, epileptische Anfälle } \\
\text { Nichtneurologisch: rheumatologische, Nieren- und Hautläsionen }\end{array}$ & $\begin{array}{l}\text { Meningitis selten } \\
\text { Lymphozytäre Pleo- } \\
\text { zytose, normal bis } \\
50 / \mu l[9]\end{array}$ \\
\hline $\begin{array}{l}\text { Granulomatose mit } \\
\text { Polyangiitis }\end{array}$ & $\begin{array}{l}\mathrm{m}=\mathrm{w} \\
\text { Auftreten nach dem 50. Le- } \\
\text { bensjahr }\end{array}$ & $\begin{array}{l}\text { Neurologisch: zerebrale Vaskulitis, Pseudotumor cerebri } \\
\text { Nichtneurologisch: Hautläsionen, Epistaxis, Pneumonien, Glomerulone- } \\
\text { phritis, c-ANCA, Anti-PR3 }\end{array}$ & $\begin{array}{l}\text { Meningitis sehr } \\
\text { selten }\end{array}$ \\
\hline
\end{tabular}

Tab. 2 Kriterien für die Diagnosestellung eines Neuro-Sweet-Syndroms. (Nach Hisanaga et al. 2005 [7])

\section{Neurologische Kriterien}

Gutes systemisches Ansprechen auf Glukokortikoide. Gelegentlich Spontanremission, jedoch oft rezidivierende (Meningo-)Enzephalitiden mit Fieber über $38^{\circ} \mathrm{C}$

2. Dermatologische Kriterien

Schmerzhafte weiche flaue erythematöse Plaques oder Knötchen vorzugsweise an Gesicht, Hals, den oberen Extremitäten und dem oberen Teil des Rumpfes auftretend

Vorwiegend neutrophile Infiltration der Dermis mit Aussparung der Epidermis und Fehlen einer leukozytoklastischen Vaskulitis

3. Weitere Kriterien

Fehlen einer kutanen Vaskulitis und Thrombose (beides dagegen häufig bei M. Behçet)

Fehlen einer Uveitis, wie sie typischerweise bei M. Behçet vorkommt

4. HLA-Assoziation

HLA-Cw1- oder B54-positiv

HLA-B1-negativ

Wahrscheinliches Neuro-Sweet-Syndrom: Alle Punkte in 1, 2 und 3 sind erfüllt.

Mögliches Neuro-Sweet-Syndrom: Neurologische Manifestation, 2 oder 4 und mindestens 1 Punkt aus 3 sind erfüllt

HLA humanes Leukozytenantigen

BSG-Erhöhung sowie eine Leukozytose. Im Liquor zeigen sich typischerweise eine leichte Eiweißerhöhung sowie eine leichte bis moderate lymphozytäre Pleozytose [3].

Die Diagnosekriterien sind in Tab. 2 dargestellt.

Der differenzialdiagnostisch zu erwägende $\mathrm{M}$. Behçet wurde im Jahr 1937 erstmalig von Hulusi Behçet [1] beschrieben. Die Erkrankung geht mit rezidivierenden oralen Aphten, rezidivierenden genitalen aphthösen Ulzerationen und rezidivierender Uveitis einher. Bei 10-30\% der Patienten kommt es im Laufe der Erkrankung zu einer neurologischen Manifestation, beispielsweise in Form eines Pseudotumor cerebri, Hirnstammlä-

\begin{tabular}{|c|c|c|}
\hline & Neuro-Sweet-Syndrom & Neuro-Behçet \\
\hline$M: F$ & $1,3: 1$ & $3,4: 1$ \\
\hline $\begin{array}{l}\text { Alter bei Erkran- } \\
\text { kungsbeginn }\end{array}$ & 30-60 Jahre & 20-30 Jahre \\
\hline $\begin{array}{l}\text { Verteilung der } \\
\text { ZNS-Läsionen }\end{array}$ & $\begin{array}{l}\text { Keine Prädilektionsstel- } \\
\text { len }\end{array}$ & $\begin{array}{l}\text { Basalganglien und } \\
\text { Hirnstamm }\end{array}$ \\
\hline Erythem & Ohne Vaskulitis & Mit Vaskulitis \\
\hline $\begin{array}{l}\text { Okulare Mitbeteili- } \\
\text { gung }\end{array}$ & $\begin{array}{l}\text { Episkleritis oder Kon- } \\
\text { junktivitis }\end{array}$ & Uveitis \\
\hline HLA-Assoziation & CQ1 und B54 & B51 \\
\hline
\end{tabular}

HLA humanes Leukozytenantigen

sionen, Hirnnervenparesen, Rückenmarksläsionen, Psychosen, Subarachnoidalblutungen und peripheren Neuropathien [12].

Bei beiden Erkrankungen handelt es sich um Multisystemerkrankungen, die mit oralen und genitalen Ulzerationen, Erythema-nodosum-ähnlichen Hautläsionen und okularen Symptomen einhergehen und das zentrale Nervensystem betreffen können.

Wesentliche Unterschiede betreffen das Manifestationsalter, die Geschlechterverteilung, die Verteilung der MRT-Läsionen, die okulare Beteiligung sowie die HLA-Assoziation und sind in Tab. 3 aufgeführt. Als ein weiteres Unterscheidungsmerkmal gilt das Fehlen einer Vaskulitis in der Hautbiopsie beim SweetSyndrom [5].

Wenn auch häufig rezidivierend, ist der Verlauf des NeuroSweet-Syndroms in der Regel benigne, die Erkrankung spricht gut auf Glukokortikoide an und hinterlässt im Gegensatz zum M. Behçet äußerst selten neurologische Langzeitschäden [3].

Ein gleichzeitiges Auftreten beider Erkrankungen ist beschrieben, ebenso Überlappungen des Neuro-Sweet-Syndroms mit anderen Autoimmunerkrankungen [2]. 
Hier steht eine Anzeige.

Springer 


\section{Therapie}

Das Neuro-Sweet-Syndrom spricht in der Regel sehr gut auf Glukokortikoide an. Üblicherweise wird mit einer Dosis von $1 \mathrm{~m} / \mathrm{kgKG}$ täglich begonnen und innerhalb von 4-6 Wochen auf $10 \mathrm{mg}$ täglich reduziert [3].

Auch Kortisonstoßtherapien über 3-5 Tage mit Methylprednisolon mit anschließender Oralisierung $(1 \mathrm{~m} / \mathrm{kgKG})$ sind in der Literatur beschrieben [2]. Ein Standard zur Länge der Glukokortikoidtherapie existiert nicht. Bekannt ist aber, dass es nach Absetzen in bis zu $28 \%$ der Fälle zu Rezidiven kommt [4]. Um dies zu vermeiden, finden sich verschiedene Therapieansätze. Eine langfristige nicht genau definierte niedrigdosierte Glukokortikoidtherapie wurde in einzelnen Fallberichten erfolgreich mit Indomethacin (100-150 mg täglich für 3 Wochen), Kolchizin (3-mal 0,5 mg täglich) oder Kalium (3-mal 300 mg täglich) kombiniert [2].

Darüber sind Fälle beschrieben, in denen die zusätzliche Gabe von Dapson, eines Antirheumatikums mit antibiotischer Wirkung, das zur Gruppe der Sulfone gehört, in einer Dosis von 75-200 mg täglich die Rezidivrate während des Ausschleichens der Glukokortikoide verringern konnte [4]. Auch hier existieren keine genauen Empfehlungen zur Therapiedauer. In 1 berichteten Fall betrug diese 6 Monate. Anschließend wurde ausgeschlichen, wobei hierzu kein konkretes Schema berichtet wurde.

\section{Fazit für die Praxis}

- Bei aseptischen Meningo-(Enzephalitiden) auf kutane Symptome achten und diese aktiv erfragen bzw. suchen.

- Das Neuro-Sweet-Syndrom spricht in der Regel gut auf Glukokortikoide an.

- Rezidive sind häufig, schwere Verläufe selten.

- Die kutane Manifestation kann der neurologischen vorausgehen oder erst verzögert auftreten. Es sind auch Fälle von gutartigen unkomplizierten steroidresponsiven Enzephalitiden mit HLA-B54-Assoziation beschrieben, bei denen trotz fehlender Hautmanifestation von einem Neuro-Sweet-Syndrom ausgegangen wurde.

- Im Gegensatz zum M. Behçet liegt eine neutrophile Infiltration der Haut ohne Vaskulitis vor.

- Das Vorliegen einer Vaskulitis oder Uveitis sollte den Verdacht Richtung M. Behçet lenken.

- Im Rahmen der Abklärung sollten neben einem großen Blutbild, den Entzündungsparametern sowie der Liquoranalyse inklusive Erregerdiagnostik auch ein autoimmunologisches Screening, eine Hautbiopsie und eine augenärztliche Vorstellung erfolgen.

- Da das Sweet-Syndrom auch tumorassoziiert sein kann, ist ein Tumorscreening zu empfehlen.

Literatur

1. Behçet $H$ (1937) Über rezidivierende, aphtöse, durch ein Virus verursachte Geschwüre im Mund, am Auge und an den Genitalien

2. Cohen PR (2007) Sweet's syndrome-A comprehensive review of an acute febrile neutrophilic dermatosis. Orphanet J Rare Dis 2:34. https://doi.org/10.1186/17501172-2-34
3. Drago F, Ciccarese G, Agnoletti AF et al (2017) Neuro sweet syndrome: a systematic review. A rare complication of Sweet syndrome. Acta Neurol Belg 117:33-42. https://doi.org/10.1007/s13760-016-0695-1

4. Fukae J, Noda K, Fujishima Ket al (2007) Successful treatment of relapsing neuroSweet's disease with corticosteroid and dapsone combination therapy. Clin Neurol Neurosurg 109:910-913. https://doi.org/10.1016/j.clineuro.2007.07.025

5. Hisanaga K (2007) Neuro-neutrophilic disease: neuro-Behçet disease and neuroSweet disease. Intern Med 46:153-154. https://doi.org/10.2169/internalmedicine. 46.0175

6. Hisanaga K, Hosokawa M, Sato N et al (1999) "Neuro-Sweet disease": benign recurrentencephalitis with neutrophilic dermatosis. Arch Neurol 56:1010. https:// doi.org/10.1001/archneur.56.8.1010

7. Hisanaga K, Iwasaki Y, Itoyama Y, the Neuro-Sweet Disease Study Group (2005) Neuro-Sweet disease: clinical manifestations and criteria for diagnosis. Neurology 64:1756-1761.https://doi.org/10.1212/01.WNL.0000161848.34159.B5

8. Maxwell G, Archibald N, Turnbull D (2012) Neuro-Sweet's disease. Pract Neurol 12:126-130.https://doi.org/10.1136/practneurol-2011-000067

9. Reske D, Petereit H-F (2004) Differenzialdiagnose chronisch-entzündlicher Erkrankungen des Zentralnervensystems: Liquordiagnostik und immunologische Parameter. Nervenarzt 75:945-952. https://doi.org/10.1007/s00115-004-1699-2

10. Sweet RB (1964) An acute febrile neutrophtlic dermatosts. Br J Dermatol 76:349-356. https://doi.org/10.1111/j.1365-2133.1964.tb14541.x

11. Tattevin P, TchamgouéS, Belem A, Bénézit F, Pronier C, Revest M (2019) Aseptic meningitis. Revue Neurologique 175(7-8):475-480

12. Uysal H, Vahaboğlu H, Inan L, Vahaboğlu G (1993) Acute febrile neutrophilic dermatosis (Sweet's syndrome) in neuro-Behçet's disease. Clin Neurol Neurosurg 95:319-322.https://doi.org/10.1016/0303-8467(93)90109-T

\section{Korrespondenzadresse}

Dr. A. Pankert

Neurologische Klinik

Städtisches Klinikum Ludwigshafen

Bremserstraße 79, 67346 Ludwigshafen, Deutschland

pankerta@klilu.de

\section{Einhaltung ethischer Richtlinien}

Interessenkonflikt. A. Pankert, M. Najaf, C. Kuen, E. Dippel und A. Grau geben an, dass kein Interessenkonflikt besteht.

Für diesen Beitrag wurden von den Autoren keineStudien an Menschen oder Tieren durchgeführt. Für die aufgeführten Studien gelten die jeweils dort angegebenen ethischen Richtlinien. Für Bildmaterial oder anderweitige Angaben innerhalb des Manuskripts, über die Patienten zu identifizieren sind, liegt von ihnen und/oder ihren gesetzlichen Vertretern eine schriftliche Einwilligung vor. 